\title{
INDAGACIÓN SISTEMÁTICA SOBRE LOS FACTORES DE PERMANENCIA Y GRADUACIÓN ESTUDIANTILES EN LA UNIVERSIDAD SURCOLOMBIANA
}

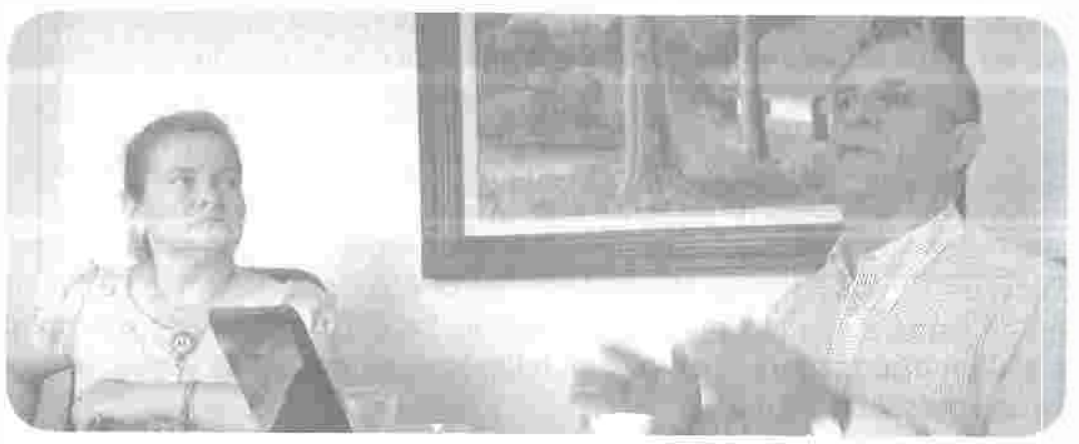

\author{
Coordinador del Proyecto: \\ Doctor Nelson Ernesto López Jiménez \\ Docente Investigador Universidad Surcolombiana \\ Equipo de trabajo - Investigadoras Grupo PACA \\ Karol Stefanía Amaya García \\ Laura Córdoba Muñoz
}

El Grupo de Investigación PACA de la Universidad Surcolombiana ha liderado el proyecto "Indagación sistemática sobre los factores de permanencia y graduación estudiantiles en la Universidad Surcolombiana" el cual hace parte del Convenio 619 de octubre de 2012 celebrado con el Ministerio de Educación Nacional.

En ese sentido, desde octubre del año 2012 el Grupo de Investigación PACA encabezado por el docente investigador Nelson Ernesto López Jiménez y las Magíster Karol Stefanía Amaya García y Laura Córdoba Muñoz han desarrollado las actividades que se definieron en el marco de la convocatoria y que responden a cada uno de los componentes que han permitido abordar la problemática de la permanencia y del abandono estudiantil en la Universidad Surcolombiana.

\section{SENSIBILIZACIÓN Y POSICIONAMIENTO DEL TEMA:}

En relación con las actividades que se propusieron para el desarrollo del componente Sensibilización y Posicionamiento del tema el 03 de octubre del 2012 se llevó a cabo una reunión con todas las directivas de la Universidad Surcolombiana en donde se socializó el Convenio 619 de 2012 (Ministerio de Educación Nacional-USCO) y el proyecto "Indagación sistematica sobre los factores de permanencia y graduación estudiantiles en la Universidad Surcolombiana".

Producto de esta reunión se elaboró la Resolución Rectoral No. 153 del 04 de octubre de 2012 por la cual se institucionaliza la Política de Permanencia y Graduación Estudiantiles en la Universidad Surcolombiana. 
Es importante destacar que el Grupo de Investigación PACA frente a la problemática del abandono asume una postura en la que el referente conceptual y teórico juega un papel trascendental dentro del análisis y estudio gue se viene desarrollando. Por ello no se hace referencia a retención y deserción, sino que se concibe la permanencia como "el proceso mediante ef cual los estudiantes que ingresan a un programa academico de pregrado egresan con la titulación respectiva" (López Jiménez: 2012). El abandono por su parte se entiende como el "acto porel cual un estudianteque cursa un programa académico decide no continuarcon sus estudios" (López Jiménez: 2012).

日 interés del equipo investigador se centro en involucrar a toda la comunidad universitaria en el desarrollo del proyecto, de tal forma gue el estudio de la problemática del abandono sea una responsabilidad de todos con el fin de que las estrategias que se implementen sean producto del trabajo colectivo en el gue docentes, directivos, estudiantes y administrativos participen de manera permanente. En ese sentido, los datos no son el referente principal sino que son un insumo para el estudio, la reflexión y el análisis de la problemática de la permanencia y graduación estudiantiles.

日 trabajo realizado permitió sensibilizar yvincular a toda la comunidad universitaria (docentes, administrativos y directivos) la cual tiene total conocimiento del proyecto y han participado en las actividades que se vienen desarrollando. La institucionalización del proyecto ha despertacto en la Universidad Surcolombiana el interés y compromiso por aunar esfuerzos para que la problemática de la permanencia y graduación estudiantiles se asuma como un tema central de las dinámicas institucionales que le competen a la Universidad Surcolombiana. Uno de los logros alcanzados a Octubre de 2013, es la expedición de la Política de Permanencia y Graduación estudiantiles en la Universidad Surcolombiana, mediante la aprobación del Acuerdo 042 del Consejo Superior Universitario, actualmente en implementación.

\section{CULTURA DE LA INFORMACION Y DE LA EVALUACIÓN DE RESULTADOS:}

Al considerar la recolección, organización y el análisis de la información relacionada con los casos de abandono estudiantil como una herramienta para abordar a problemática del abandono en la Universidad Surcolombiana, el equipo investigador consideró pertinente la realización de reuniónes de trabajo con el Personal responsable de Bienestar Universitario y la jefe del Centro de Tecnología de a Información y Comunicación CETIC para conocer las dinámicas de cada una de estas dependencias, los procesos que lideran y la forma como estos son determinantes para b permanencia y la graduación estudiantires.

Durante esta sesion de trabajo los resultados obtenidos fueron los siguientes:

1. Se conocio el proceso mediante el cual el Centro de Tecnología de Información y Comunicación de la Universidad Surcolombiana reporta información sobre los estudiantes al Sistema para la Prevención de la Deserción de la Educación Superior - SPADIES. El cual consiste en que cada dependencia académica y administrativa hace entrega semestralmente de un reporte en el que se especifica el comportamiento de los estudiantes. Una vez presentado el reporte por cada dependencia, el Centro de Tecnología de Información y Comunicación de la Universidad Surcolombiana consolida la información y la reporta ante el SPADIES teniendo en cuenta los requerimientos exigidos por el sistema. 
2. Se discutió y analizó el proceso que se realiza en la Universidad Surcolombiana para re. unir la información que se debe reportar al Sistema para la Prevención de la Deserción de la Educación Superior-SPADIES, en donde se encontró que algunos docentes de los programas académicos de $\mathrm{b}$ Universidad no cumplen con el compromiso de reportar toda la información solicitada en el tiempo asignado para tal fin.

3. Dentro de las formas y procedimientos que realiza el Centro de Tecnología de Información y Comunicación - CTIC de la Universidad Surcolombiana para dar a conocer el comportamiento sobre permanencia y graduación estudiantiles a todas las dependencias de la Universidad, se estableció conocer que la directora de CTIC mediante reuniones de socialización en las Facultades, daba a conocer dicha información; no obstante ante la falta de compromiso e interés por parte de los docentes y administrativos fue suspendida esta actividad. Dicha situación se presentó porque algunos docentes se muestran poco interesados con este tipo de actividades debido a que consideran que los funcionarios administrativos no son autoridades académicas para hacer referencia a estas situaciones que son propias de los docentes y los estudiantes.

Frente a esta situación el equipo de trabajo preciso la necesidad de diseñar una estrategia que permita consolidar un Sistema de Información sobre los procesos de permanencia y graduación estudiantil a todas las dependencias de la Universidad Surcolombiana.

Además de lo anterior, el equipo investigador en alianza con los Consejos de Facultad, b Vicerrectoría Académica, los docentes, estudiantes, el Centro de Tecnología de la Información y Comunicación y la Oficina de Planeación avanzó en l estructuración de siete informes, uno por cada Facultad (ingeniería, Educación, Derecho, Salud, Ciencias Sociales y Humanas, Ciencias Exactas y Economía y Administración) en donde se describe los componentes teleológicos de los programas de pregrado de las siete facultades, su historia y las actividades de investigación y proyección social que se adelantan en cada uno de ellos. Posteriormente, se hizo un análisis de los datos relacionados con el número de estudiantes matriculados y abandonadores durante los periodos 2008-2009-2010-2011-2012 en donde a través de gráficos de barras y líneas se evidencia el comportamiento de cada programa académico de pregrado. Así mismo, junto con la Oficina de Planeación se recolectó y organizó $\mathrm{b}$ información pertinente que permitieron determinar los costos del abandono por Facultad y por Programa.

Como resultado de este trabajo de caracterización, se adquirió con la Univerșidad Tecnológica de Pereira un Programa de Alertas Tempranas el cual se convierte en un instrumento guía para la entrevista individual que se realiza a los estudiantes que ingresan a primer semestre, cuya finalidad es identificar anticipadamente algunos factores de riesgo que puedan dificultar la permanencia del estudiante en la educación superior.

\section{ME JORAMIENTO DEL RENDIMIENTO ACADÉMICO Y DE LA ARTICULACIÓN ENTRE NIVELES}

El Programa de Actualización y Capacitación Académica Profesoral P.A.C.A.P desarrolló un proceso de fundamentación, actualización y capacitación directamente relacionado con la apropiación de nuevas estrategias y habilidades curriculares, pedagógicas, evaluativas, investigativas y tecnológicas, que respondan a los retos derivados de la sociedad del conocimiento y de las emergencias concretas de los procesos de globalización y mundialización actuales. Quienes conformaron el equipo de trabajo fueron los Decanos de Facultad de los programas académicos, directivos administrativos de la Universidad Surcolombiana, rectores de las instituciones de educación media y delegados de las alcaldías municipales. 
La propuesta del P.A.C.A.P. consistió en la realización de cinco talleres de formación relacionados con los siguientes temas - problemas: Formación por competencias, Docencia e investigación Universitaria, Evaluación del rendimiento académico, Modernización académica y curricular: integración e interdisciplinariedad y los Impactos de las TICS como herramientas de aprendizaje.

Cabe destacar que el interés por desarrollar los talleres mencionados anteriormente radica en la necesidad de contribuir con la formación de los docentes de la Universidad Surcolombiana ya que ellos juegan un papel determinante tanto en el abandono como en la permanencia y graduación estudiantiles dependiendo de las estrategias que utilicen para el desarrollo de sus cursos y la formación de los estudiantes.

En relación con los procesos de articulación con la Educación Media el equipo investigador logro que la Universidad Surcolombiana firmara convenios de cooperación con cuatro (4) Instituciones de Educación Media del Departamento del Huila, de las cuales dos son del municipio de Neiva y una del municipio de Pitalito y de La Plata.

Igualmente, se estableció comunicación y contacto con las alcaldías de los municipios de Neiva y Pitalito los cuales son conocedoras del Convenio ó 19 de 2012, el proyecto investigativo que se viene adelantando, las actividades que hacen parte de este y el compromiso adquirido por la Universidad Surcolombiana en pro de increme ntar la permanencia y graduación estudiantiles. Estos encuentros han logrado concientizar y comprometer a las autoridades locales en relación con la importancia de apoyar los procesos que actualmente desarrolla la Universidad Surcolombiana para ofrecer a sus estudiantes un Programa lntegrado de Estímulos y Servicios (PIES) y actualmente se están programando las reuniones. para protocolizar la firma de los mismos.

\section{FORTALECIMIENTO DE PROGRAMAS DE FOMENTO A LA PERMANENCIA:}

Considerando ef fortalecimiento de programas de fomento a la permanencia y graduación estudiantiles como una estrategia esencial que favorece a los estudiantes y que contribuyen con su proceso de formación profesional se consideró de vital importancia avanzar en b evaluación de los actuales programas que Bienestar Universitario ofrece la Universidad Surcolombiana.

Para llevar a cabo este proceso se inició con la revisión de los documentos de carácter normativo que existen en la institución para regular y soportar los servicios que se of recen en el Area de Bienestar Universitario. Posteriormente, se evaluó el portafolio de servicios, ef número de beneficiados y los recursos financieros que se destinan anualmente para el ofrecimiento de los servicios y el desarrollo de las actividades que lidera el Área de Bienestar Universitario.

Además como uno de los productos de este trabajo desarrollado se adquirió un Programa de Orientación Profesional POP con la UniversidadTecnológica de Pereira el cual tiene como principal motivación ayudar a los jóvenes a evaluar sus posibilidades en términos de las actitudes y aptitudes frente a una posible carrera, es decir, que quiere estudiar y qué puede estudiar. É programa proporciona al estudiante una aproximación del conocimiento que él tiene sobre sí mismo, sus capacidades, su rendimiento, sus motivaciones e intereses, su inteligencia, aptitudes y su personalidad. Estos elementos son el punto de partida para tomar una decisión certera acorde a lo que le interesa, lo que puede y cómo se visualizarä en un escenario futuro desempeñándose en una profesión.

Un avance importante a destacar es la creación del Comité Instituciona I para el Fomento de la Política de Permanencia y Graduación Estudiantiles (24 de febrero de 2014), que garantizara el desarrollo concreto de este proceso en las diferentes Facultades y Programas que constituyen el portafolio académico de la Universidad Surcolombiana. 